\title{
ELECCIONES LOCALES EN GALICIA 2007: LA CONSOLIDACIÓN DEL CAMBIO DE CICLO POLÍTICO*
}

\author{
POR \\ ÁLVARO XOSÉ LÓPEZ MIRA \\ Profesor Titular de Ciencia Política y de la Administración \\ Universidad de Vigo
}

\section{INTRODUCCIÓN}

En el interior de la Comunidad gallega, la mayor parte de las inquietudes políticas previas al 27 de mayo, se traducían en el posible aval que podrían otorgar los electores al Gobierno de coalición entre el PSdeG-PSOE y los nacionalistas del Bloque Nacionalista Galego (BNG) en el ecuador de su mandato autonómico. Más que nunca, el peso económico, demográfico, político-social y simbólico de las siete grandes ciudades gallegas (Vigo, A Coruña, Ourense, Santiago de Compostela, Lugo, Pontevedra y Ferrol) centró los cuerpos de elite de los partidos de cara a la batalla electoral que se avecinaba. El resto de las villas y pueblos de Galicia quedaron reducidos, en los titulares mediáticos y encuestas de opinión, a aquellos en los que se debatían escándalos urbanísticos, penales o con antecedentes de transfuguismo de los candidatos; es decir, apenas existieron. De otro lado, las confrontacio-

* La práctica totalidad de los datos proceden de la página web relativa a estas elecciones del Ministerio del Interior. Los cuadros son de elaboración propia. 
nes extra-gallegas parecían no afectar demasiado al electorado de esta Comunidad Autónoma tras el fiasco de la reforma estatutaria marcado, al parecer, por los órganos centrales del Partido Popular contra el criterio de su franquicia en Galicia que, sin embargo, acabó acatando la decisión superior unos meses antes de estos comicios locales.

Los electores gallegos acabaron diseñando un triángulo escaleno casi perfecto entre la terna de fuerzas políticas representadas en el Parlamento autonómico, con porcentajes (redondeados) del $40 \%$ de votos para el Partido Popular, $30 \%$ para el PSdeG-PSOE y $20 \%$ para el BNG, confirmando un escenario a tres cada día más asentado, y cuyo desglose veremos a continuación analizando los principales datos.

\section{LOS RESULTADOS}

Un comentario como este no puede descender a la microescala municipal que exigiría visualizar los resultados en cada uno de lo trescientos quince ayuntamientos gallegos, por lo que nos limitaremos a ofrecer datos globales en votos, concejales, cifra de instituciones locales ${ }^{1}$ gobernadas por los distintos partidos y, eso sí, resultados algo más detallados de las antedichas siete ciudades gallegas, sin olvidar las cuatro Diputaciones provinciales.

Con una abstención del $37 \%$, algo más de tres puntos superior a las elecciones locales de 2003, muy semejante a la media española, el Partido Popular continuó siendo la fuerza política más votada con 656.000 sufragios (baja dos puntos porcentuales en relación a 2003), pero superada ampliamente por los dos coaligados en los gobiernos locales $^{2}$ desde los anteriores comicios que sumaron cerca de 800.000 apoyos: 479.000 el PSOE, 316.000 el BNG, incrementando el primero los dos puntos porcentuales que pierde el PP. La diferencia lograda por los socios de gobierno que había sido de 83.000 votos en 2003 , ha pasado a cerca de 140.000 , un $8 \%$ del total de sufragios emitidos por los gallegos y un $10 \%$ del conjunto de apoyos ciudadanos recibidos, en la literaria expresión de Manuel Rivas, por la trinidad política gallega ${ }^{3}$.

${ }^{1}$ Excluiremos las nueve Entidades Locales menores registradas en Galicia, ocho en la provincia de Pontevedra y la otra en la de Ourense.

2 En 2005, las mismas fuerzas formaron gobierno de coalición en la Xunta de Galicia al lograr entre ambas mayoría absoluta de escaños en el Parlamento de la Comunidad.

${ }^{3}$ De efectuarse una extrapolación de estos resultados a una convocatoria autonómica, el Partido Popular perdería dos escaños que recibiría el Bloque Nacionalista 
Estos datos globales otorgaron al Partido Popular un total de 1.789 concejales (2.042 cuatro años antes), 1.117 al PSdeG-PSOE (frente a 927 en 2003) y 661 a los nacionalistas del BNG (595 en 2003). Las restantes fuerzas políticas (incluidas las prolíficas, en este tipo de comicios, candidaturas independientes, muchas de ellas fruto de escisiones de los grandes partidos), sumaron una cifra de 280 concejales, de los que cabría destacar los 66 de Terra Galega (43 de ellos en la provincia de A Coruña, 10 de los cuales en el municipio de Narón). Curiosamente, a pesar de la diferencia en sufragios populares, la cifra conjunta de concejales de los socios de gobierno no superó a los logrados por el partido más votado, lo cual es revelador de dónde obtuvieron sus apoyos unos y otro, en los segmentos demográficos de los diversos municipios: el PP tendrá muchos representantes en muchos municipios pero, sobre todo, en los más pequeños. De hecho, 152 candidatos de este partido, alcanzaron la mayoría absoluta en sus respectivos concejos (es decir, casi la mitad de los 315 ayuntamientos gallegos), por solamente 39 mayorías absolutas del PSOE y 8 del BNG. Tras la constitución de las Corporaciones municipales (a falta de cuatro pendientes de recursos), el PP gobernará 160 municipios, el PSOE 102, el BNG 32, Terra Galega 4 y candidaturas independientes los 13 restantes.

En las siete grandes urbes gallegas desaparecieron todas las mayorías absolutas, siendo el Partido Popular la candidatura más votada en cuatro de ellas y el PSOE en las tres restantes. Veamos los datos desglosados.

\begin{tabular}{|l|c|c|c|}
\hline A CORUÑA & Votos & Porcentaje & Concejales \\
\hline PSOE & 41.285 & $35,02 \%$ & 11 \\
\hline PP & 37.085 & $31,46 \%$ & 10 \\
\hline BNG & 24.355 & $20,66 \%$ & 6 \\
\hline
\end{tabular}

Contra lo apuntado en las encuestas y con la abstención más elevada de las ciudades gallegas, más del $46 \%$, el PSdeG-PSOE perdió con nitidez la hegemonía electoral que mantenía en esta ciudad en elecciones locales (y sólo en ellas) desde 1983, aunque en 2003 únicamente un puñado de votos le había permitido a Francisco Vázquez lograr mayoría absoluta con algo más del $45 \%$ de sufragios y varios cientos de votos

Galego, reafirmando la mayoría absoluta de las dos fuerzas políticas progresistas en la Cámara gallega. 
menos que los otros dos partidos con representación en el consistorio coruñés. Su otrora teniente de alcalde mantiene, sin embargo, la condición de lista más votada ante la subida de tres concejales del PP, con nuevo y contestado candidato ${ }^{4}$, Carlos Negreira, y el mantenimiento de la cuota representativa del BNG, con el candidato inamovible de las seis últimas convocatorias. Tal parece que el sector de votante conservador que apoyaba al anterior alcalde, no confió tanto en su sucesor Javier Losada y, de hecho, las patéticas reclamaciones del representante diplomático español en el Vaticano para que pactase con el PP se repitieron hasta la saciedad siendo, lógicamente, desoídas.

\begin{tabular}{|l|c|c|c|}
\hline FERROL & Votos & Porcentaje & Concejales \\
\hline PSOE & 11.852 & $32,33 \%$ & 9 \\
\hline PP & 9.409 & $25,66 \%$ & 7 \\
\hline EU-IU $^{5}$ & 5.203 & $14,19 \%$ & 4 \\
\hline IF $^{6}$ & 4.675 & $12,75 \%$ & 3 \\
\hline BNG & 3.219 & $8,78 \%$ & 2 \\
\hline
\end{tabular}

El ayuntamiento más diverso políticamente de los siete grandes, modificó sus preferencias políticas que, en 2003, le habían otorgado el mandato a una coalición del PP (7 concejales) con IF (6 concejales). En 1999, sin embargo, había tenido alcalde de un BNG (8 concejales entonces) que, en estos comicios, alcanzó sus peores resultados en las urbes gallegas (desciende de 6 a 2 concejales). Con una abstención también elevada ${ }^{7}$ de más del 44\%, EU-IU, con una candidata líder de la oposición a la planta industrial de Reganosa, duplicó sus actas y el PSOE, con un nuevo candidato, las elevó de 4 a 9, lo que ha permitido un pacto político para el gobierno ferrolano (PSOE-IU) absolutamente inédito en el resto de Galicia ${ }^{8}$. Esta circunstancia de zigzagueante cambio político, aparentemente errático, al margen de cualesquiera otras

${ }^{4}$ De hecho, el anterior líder local presentó candidatura propia.

${ }^{5}$ Esquerda Unida-Izquierda Unida.

${ }^{6}$ Independientes de Ferrol.

${ }^{7}$ La abstención es más alta en las siete urbes gallegas que en el conjunto de Galicia.

${ }^{8}$ EU-IU logró en el conjunto de Galicia 14 actas de concejal (la mayoría en la comarca ferrolana) con el 1,37\% de los sufragios y ninguna otra en las restantes seis ciudades de la Comunidad. 
consideraciones de índole ideológica, obedece en buena medida al sentimiento de frustrante e inevitable decadencia que sobrevuela una ciudad privada de su destino histórico como centro militar, que además sufrió una reconversión industrial traumática, perdió su estela de bienestar económico hasta agonizar demográficamente (ya es la más pequeña de las siete urbes) y, como colofón hasta el momento, se ve asolada por la sensación de riesgo físico y consiguiente conflicto social que está provocando una industria peligrosa instalada en sus orillas, de manera imperativa y con notorias irregularidades, por el anterior Gobierno de la Xunta, a pesar de la irracionalidad - no discutida por nadie, paradójicamente, una vez que ha cambiado el signo de ese Gobierno- de tal emplazamiento.

\begin{tabular}{|l|c|c|c|}
\hline $\begin{array}{l}\text { SANTIAGO DE } \\
\text { COMPOSTELA }\end{array}$ & Votos & Porcentaje & Concejales \\
\hline PP & 18.664 & $39,01 \%$ & 11 \\
\hline PSOE & 18.269 & $38,19 \%$ & 10 \\
\hline BNG & 7.871 & $16,45 \%$ & 4 \\
\hline
\end{tabular}

Con una abstención del $43 \%$, los electores compostelanos inviertieron un concejal que situó al PP y un nuevo candidato ${ }^{9}$ como fuerza más votada ante el PSOE del alcalde Xosé Bugallo, manteniendo a un BNG, con fuertes discrepancias internas a nivel local ${ }^{10}$, en su anterior posición. Los tradicionales buenos resultados de los socialistas en la capital de Galicia (también aquí exclusivamente en comicios locales), se vieron en esta ocasión matizados por los electores de esta urbe ${ }^{11}$, quienes consiguieron confundir a todas las encuestas electorales (incluida la

\footnotetext{
${ }^{9}$ En realidad, los cambios son constantes en los sucesivos candidatos a la alcaldía del PP, también sucesivamente derrotados.

${ }^{10} \mathrm{El}$ candidato fue rechazado en votación por la mayoría de la militancia aunque, finalmente, se le permitió presentarse.

${ }^{11}$ Cabría anotar aquí que los municipios de la periferia compostelana han elevado sus cifras de población de manera paralela al enorme desarrollo de nuevas urbanizaciones (los altos precios de la vivienda del municipio central, por el contrario, han estancado la de Santiago que no consigue alcanzar los cien mil residentes) y cambiado sus clásicos electorados rurales de forma drástica ante el incremento de población más joven que trabaja en la ciudad central y reside en los municipios próximos, hasta el punto de que el PP ha perdido sus más clásicos bastiones en la comarca, como los ayuntamientos de Ames (PSOE) o Teo (BNG).
} 
del CIS) que apuntaban a una mayoría absoluta alcanzable por parte del PSdeG-PSOE.

\begin{tabular}{|l|c|c|c|}
\hline LUGO & Votos & Porcentaje & Concejales \\
\hline PSOE & 23.375 & $45,04 \%$ & 12 \\
\hline PP & 18.428 & $35,51 \%$ & 9 \\
\hline BNG & 7.417 & $14,29 \%$ & 4 \\
\hline
\end{tabular}

Con la abstención más baja de las urbes gallegas $\left(35,5 \%{ }^{12}\right)$, un alcalde con estela ascendente que venía de una mayoría absoluta con más del $51 \%$ de los votos (13 concejales) y todas las encuestas pronosticando un previsible incremento de esa mayoría, la pérdida de la misma por parte del candidato López Orozco supuso una sorpresa de tal calibre que el propio candidato amenazó con abandonar el partido si le obligaba a pactar con sus otrora (1999-2003) socios del BNG, partido que incrementó el concejal perdido por el PSOE. Los seis puntos porcentuales que descendió esta fuerza, se repartieron a partes iguales entre PP y BNG, lo que revela un cierto voto de castigo al candidato socialista.

\begin{tabular}{|l|c|c|c|}
\hline OURENSE & Votos & Porcentaje & Concejales \\
\hline PP & 26.160 & $42,25 \%$ & 13 \\
\hline PSOE & 16.428 & $26,53 \%$ & 8 \\
\hline BNG & 12.253 & $19,79 \%$ & 6 \\
\hline
\end{tabular}

Con un nuevo candidato auspiciado por el todopoderoso Presidente de la Diputación provincial frente al anterior alcalde, el Partido Popular perdió la mayoría absoluta de la que había disfrutado en las tres legislaturas previas, en un marco de abstención casi idéntico al de

${ }^{12}$ Aún así, incrementada en cinco puntos respecto a la convocatoria de 2003. La participación en elecciones locales en la provincia de Lugo ha superado desde la década de los ochenta la media gallega (moviéndose Ourense en parámetros similares) e, incluso en varias ocasiones, la media española, interesante dato que escapa de nuestro objeto de análisis en este artículo, pero que cabría relacionar con la desmesurada influencia política que juegan en estas dos provincias ambas Diputaciones provinciales y sus pretendidamente carismáticos y, hasta ahora aparentemente incombustibles, líderes. 
2003. EI PSdeG-PSOE dio el sorpasso hacia la segunda posición que ocupaba el BNG desde 1999 y el pacto de gobierno entre ambas fuerzas políticas, también las dos con candidatos renovados, ha minimizado incluso el triunfo del PP en el conjunto provincial (por tanto, en la Diputación), ya que la capital tiene un peso económico, demográfico y político desmesurado en un territorio envejecido que avanza rápidamente cara a la despoblación en buena parte del mismo.

\begin{tabular}{|l|c|c|c|}
\hline VIGO & Votos & Porcentaje & Concejales \\
\hline PP & 66.574 & $44,04 \%$ & 13 \\
\hline PSOE & 44.563 & $29,48 \%$ & 9 \\
\hline BNG & 28.116 & $18,60 \%$ & 5 \\
\hline
\end{tabular}

También en la urbe más populosa de Galicia se incrementó en tres puntos la abstención del año 2003 y, en una ciudad compleja que tradicionalmente eludió las mayorías absolutas, el electorado concentró sus concejales en sólo tres fuerzas políticas, eliminando la presencia del primer alcalde de la democracia, Manuel Soto (2 concejalías) y su perenne candidatura, consiguiendo el Partido Popular y su candidata y alcaldesa en franca minoría (10 de 27), Corina Porro, algo más del $10 \%$ de los apoyos de su partido en la Comunidad y una subida de tres escaños y once puntos porcentuales, a pesar de no lograr la mayoría absoluta y, por consiguiente, perder la alcaldía. Obviamente, la traumática ruptura del pacto PSOE-BNG a los pocos meses de su suscripción y que había propiciado la investidura por mayoría absoluta de Ventura Pérez Mariño en 2003, pasó una factura considerable a ambas fuerzas (especialmente a la segunda que perdió dos concejalías y seis puntos porcentuales) no obstante haber renovado sus respectivos candidatos a la alcaldía con personas, Abel Caballero y Santiago Domínguez Olveira, que habían ocupado altos cargos (Autoridad Portuaria de Vigo y Secretaría General para el Deporte) desde el establecimiento del nuevo Gobierno bipartito de la Xunta en 2005.

\begin{tabular}{|l|c|c|c|}
\hline PONTEVEDRA & Votos & Porcentaje & Concejales \\
\hline PP & 19.387 & $44,15 \%$ & 12 \\
\hline BNG & 12.412 & $28,26 \%$ & 7 \\
\hline PSOE & 9.807 & $22,33 \%$ & 6 \\
\hline
\end{tabular}


La única de la siete ciudades gallegas en la que los nacionalistas del BNG eran la fuerza más votada, a punto estuvo de suponer una sorpresa $^{13}$, inesperada incluso hasta para un Partido Popular que había aupado como candidato a una persona totalmente ajena a Pontevedra (era alcalde de la villa turística de Sanxenxo), promotor de profesión (con intereses directos en la capital provincial en unos momentos de alta sensibilidad política y social en este tema), quien confesó que financiaba él la campaña y que, desde luego, no contaba con el beneplácito de los correligionarios locales y la anterior candidata del Partido Popular a la alcaldía pontevedresa (al menos en un primer momento). Pese a todo ello, con unos índices de abstención del 39,49\% (tres puntos más que en 2003), aumentó dos concejales y cerca de nueve puntos porcentuales la anterior representación de su partido. El Bloque $\mathrm{Na}$ cionalista Galego y su candidato, Miguel Anxo Fernández Lores, alcalde los últimos ocho años, aunque mantuvo la alcaldía, perdió tres concejales y más de diez puntos porcentuales con respecto a 2003 y, por su parte, el PSOE, con la candidata y teniente de alcalde, Teresa Casal, incrementó en más de cuatro puntos porcentuales y una concejalía su anterior representación, lo que ha permitido reeditar el pacto de gobierno en la ciudad pontevedresa otros cuatro años.

Un último resultado que queríamos apuntar en esta sumaria crónica es el relativo a las cuatro Diputaciones provinciales gallegas. Estos organismos son objeto de acerba crítica por su inadaptación y alteridad respecto del modo de ser de la convivencia local en Galicia desde el mismo instante en que se instauró la tetrapartición liberal de su territorio en 1833, y no sólo por parte de los doctrinarios y tratadistas gallegos, sino por iuspublicistas como Morell, Parada, Baena y otros. Por no remontarnos demasiado lejos en el tiempo, cabría reseñar cómo en la transición y a lo largo del proceso de elaboración y aprobación del vigente Estatuto de Autonomía de Galicia de 1981, los poderes provinciales fueron absolutamente decisorios en la inicial configuración cercenada (provincialista se ha denominado) de la autonomía gallega; posteriormente, se convirtieron en auténticos feudos electorales (de UCD, primero, del PP — con matices_-, después), que con la sobrerrepresentación que Lugo y Ourense tienen en el Parlamento gallego ${ }^{14}$, las

${ }^{13}$ Un conocido analista político llegó a afirmar que en Pontevedra el electorado había castigado la prolífica gestión del gobierno local. De hecho, este resultado también pulverizó las previsiones de las encuestas.

${ }^{14}$ Los aproximadamente 650.000 habitantes de ambas provincias suman 29 escaños en el Parlamento autonómico, A Coruña, con más de 1.100.000 se ha de conformar con 24 y Pontevedra, con más de 900.000, con 22. 
hicieron convertirse en elementos imprescindibles para configurar las entonces balbuceantes instituciones del poder autonómico, el cual, en todas las ocasiones que intentó limitar los excesos de estos organismos (disposición estatutaria - adicional tercera- que prevé imperativamente la coordinación de sus actividades y la unión de sus presupuestos con el de la Xunta, Ley de Coordinación de Diputaciones de 1989, intento de establecer las comarcas como entidades locales territoriales a partir de ese mismo año, etc.), se topó con un muro hermético que impidió el más mínimo atisbo de modernización política de estas malhadadas instituciones, un auténtico contrapoder opuesto, en no pocos casos, a unas directrices globales para Galicia (desde la ordenación del territorio hasta el régimen local). En fin, qué decir de la complicidad en esta situación de una legislación estatal que consiente aún hoy que sean las entidades territoriales más alejadas de cualesquier criterio de racionalidad, eficiencia, objetividad, e incluso control externo ${ }^{15}$, etc. en el gasto de los sustanciosos fondos públicos que administran ${ }^{16}$ a través de recursos financieros que no recaudan y que le transfieren otras administraciones $y$, finalmente, un sistema electoral que permite elegir a sus miembros de manera material y formalmente indirecta (en segundo grado los diputados provinciales, en tercer grado el Presidente de la Diputación), sin que los electores sepan siquiera quiénes son los candidatos a ocupar tales cargos públicos electos, tal parece, que siguiendo un modelo propio del primer liberalismo decimonónico (recuérdese, no precisamente democrático), como los diputados en Cortes de la Constitución de 1812.

Añadamos que el hecho de que los distritos para estos comicios sean los partidos judiciales utilizados en 1979 (provenientes de la división de 1965), vulnera sin tapujos la exigencia de proporcionalidad y el principio de igualdad de voto con tan hondo fundamento constitucional y legal, por no hablar de la palpable renuencia que tanto al constitu-

${ }^{15}$ El Consello de Contas de Galicia se ha quejado amargamente de la carencia absoluta de instrumentos coercitivos que tiene ante la actitud sistemáticamente incumplidora en materia de fiscalización externa de ciertas Diputaciones.

${ }^{16}$ Por aportar un único dato, el presupuesto anual de las Diputaciones gallegas triplicó el año pasado el del mayor ayuntamiento gallego, superando incluso el de la poderosa Consellería de Política Territorial. Las razones de por qué la Diputación de Lugo - que lleva años ejecutando sólo la mitad- supera el presupuesto de la de Pontevedra (con el triple de habitantes) y más que duplica el de la ourensana (con un cifra similar a Lugo, pero endeudada cerca del límite legal del 110 por ciento de su presupuesto), no parecen fácil de explicar sólo con criterios racionales de mera lógica poblacional (como sí rige para los ayuntamientos), ni tampoco, desde luego, se justifica al amparo del inefable interés provincial. 
yente como al legislador le merecen el sufragio indirecto. Todo ello permite hechos como el de que el Partido Popular haya obtenido el $52 \%$ de los escaños de la Diputación pontevedresa (14 de 27) con el $42 \%$ de los sufragios y unos 25.000 votos menos que la suma PSOE-BNG. Pero esto no resulta excepcional; en la anterior convocatoria de 2003 , la misma fuerza política logró el $56 \%$ de los diputados provinciales ( 15 de los 27) sin llegar al $40 \%$ de los votos, 37.000 menos que el conjunto PSOE-BNG. También con algunos miles de votos menos que el citado tándem partidario en 1999, el PP alcanzó los 16 escaños en la Diputación pontevedresa (el $60 \%$ de los mismos). En fin, huelga señalar que primas electorales de entre 10 y 16 puntos, resultan difícilmente cohonestables con el conjunto de nuestra regulación electoral y puede coadyuvar a introducir todavía más confusión en una ciudadanía que ya lo tiene bastante complicado para entender un modelo conscientemente abstruso. A pesar de perder dos diputados, en Ourense el Partido Popular conservó su mayoría absoluta (14 de 25 con un $47 \%$ de voto) $y$, sin embargo, perdió la de Lugo (con el mismo porcentaje de voto, curiosamente, que el obtenido en Pontevedra) a manos de la coalición que suma 14 escaños frente a 11. En A Coruña, ya gobernada por un bipartito PSOE-BNG desde 2003, estas fuerzas mejoraron sus resultados pasando de 16 a 18 escaños (de un total de 31 ).

\section{ALGUNAS REFLEXIONES SOBRE LOS RESULTADOS}

En nuestra mente se agolpan más de las permisibles en un texto de esta índole, por lo que nos limitaremos a señalar unas pocas de tipo considerablemente genérico. En primer lugar, parece evidente que los electores gallegos apoyaron, de manera bastante sustancial, el cambio a nivel local del monolitismo partidario que caracterizó la política gallega de los últimos lustros y que quebró en 2005. La prueba más palmaria es que, pese a la fortaleza electoral del Partido Popular de Galicia, todas las grandes ciudades, sus entornos periurbanos y la mayoría de las villas medias cabeceras de comarca, estarán gobernadas por la coalición de nacionalistas y socialistas. Aunque mantuvo más de la mitad de las alcaldías, sólo una cuarta parte de la población gallega tendrá gobiernos locales del PP, lo que indica bien a las claras el agotamiento temporal de su propuesta de gobierno y, consiguientemente, la necesidad de renovarlo. Uno de los pocos síntomas que permiten vislumbrar que este proceso ha podido ya comenzar, es el hecho de que en las siete ciudades, el PP ha aumentado su peso electoral de 67 a 75 concejales (casi todos a costa de las correlativas bajadas del BNG) y, con anterioridad, estas urbes han sido indicadores anticipados de cambios 
de tendencia de voto a nivel global de Galicia, no cataclísmicos o repentinos, sino pausados y progresivos. Cosa distinta es que este mismo partido haya perdido las tres alcaldías que ostentaba como consecuencia de un ensimismamiento político casi autista (quizás consecuencia epigonal de su apabullante fortaleza político-electoral en los últimos tres lustros), que lo ha incapacitado para pactar con otras fuerzas, a pesar de sus denodados esfuerzos postelectorales - dirigidos esencialmente al BNG - que, obviamente, llegaron demasiado tarde y rozaron, en algún caso, el ridículo ${ }^{17}$.

Enlazando con lo apuntado, la segunda reflexión nos llevaría a reseñar el hecho de que el sistema de partidos en Galicia es lo suficientemente diferente del general español; tanto como para permitirnos afirmar que las estrategias que a nivel general (o de algún territorio) podrían ser beneficiosas para un partido de ámbito estatal pueden, simultáneamente, resultarle perjudiciales en algún otro territorio. $Y$ esto ha coadyuvado a impedir que el Partido Popular de Galicia tuviese la menor posibilidad de pactar con las otras grandes fuerzas del espectro político gallego pese a que, en algunos aspectos, tal vez esté más próximo a BNG y/o PSOE, que estos entre sí18. En nuestra opinión, sería un síntoma de normalidad política en una democracia consocional y representativa con frecuencia altamente proporcional, y probablemente también signo de enriquecimiento de la cultura política, que el abanico de pactos fuese algo más abierto que el posibilitado por el enrarecido panorama político español actual. De todas formas, sin pretender pontificar, no parece de recibo que frívolos dirigentes políticos del signo que sean, carentes de cualquier resabio de prudencia, pongan en entredicho las normas que rigen el proceso electoral cuando los resultados finales no son los deseados por ellos. Ni dichas normas, ni su respaldo constitucional, deben ser juguete del capricho de aquéllos cuando ya se ha dirimido el enfrentamiento en las urnas que, no se olvide, es un procedimiento pacífico de encauzar el conflicto permanente que supone la política, porque al ser el resultado electoral aceptado por todos, vencedores y vencidos, se destierran los mecanismos violentos

${ }_{17}$ Votando sus concejales electos al cabeza de lista del BNG aunque éste no optase a la alcaldía.

${ }^{18}$ De hecho, los pactos locales no resultaron nada sencillos a nivel desagregado, a pesar del acuerdo marco autonómico (que tampoco lo fue). No se olvide que PSdeG y BNG fueron y son competidores por semejante espacio político, lo que de suyo los debilita frente al supuesto adversario común, el Partido Popular, quien sistemáticamente resultó beneficiario de tal rivalidad hasta los grandes pactos de 2003 y 2005. 
de resolución de dicho conflicto. Como tales normas jurídicas, podrán ser discutidas y modificadas en la sede correspondiente (con el máximo consenso posible si hablamos de normativa electoral), pero vituperarlas una vez aplicadas (de manera exactamente igual para todos los contendientes), recuerda aquellos tiempos infames de nuestra historia política en los que las constituciones y las leyes se aprobaban como instrumentos de las facciones de turno contra sus rivales políticos: el siguiente paso lógico, si se insiste en caminar por tan espinoso sendero, sería el de prescindir de las constituciones y de las leyes.

En tercer y último lugar, convendría apuntar de modo destacado, el hecho de que una buena parte de los electores gallegos ha rehuido, en las agregaciones de sus preferencias políticas a través del voto, de otorgar plácidas mayorías absolutas en las grandes corporaciones municipales de la Comunidad ( $y$, como hemos señalado, este voto puede tener algo de premonitorio). EI PSOE las ha perdido en Lugo y A Coruña y el PP en Ourense; pero, además, el BNG ha dejado de ser la fuerza más votada en Pontevedra, el PSOE en Santiago de Compostela y el PP en Ferrol; el caso de Vigo admite otras variables por la peculiar forma en la que accedió a la alcaldía el PP, como ya hemos explicado. Diversas son las lecturas que se pueden extraer de tales datos. La más obvia brota inmediatamente: a los ciudadanos aparenta no molestarles la cultura pactista contra la que braman algunos dirigentes políticos; antes al contrario, parecen fomentar las debilidades de los actores o la competencia entre ellos en vez del monopolio. Aunque sólo sea reflejo de una circunstancia coyuntural, podría pensarse que se pretende buscar la eficacia en la gestión pero liberándola de engreídos caudillismos, tan fáciles de alimentar en unos niveles locales plagados de medianías. Otra inferencia, menos evidente $y$, sin duda, más personal, podría aventurarnos en la hipótesis de que el descrédito de la política y sus principales actores que patentizan, sin excepción y muy claramente, las últimas encuestas periódicas del CIS (fenómeno sumamente preocupante en nuestra opinión, pues el desprestigio de la clase política acabará afectando, sin duda alguna, al propio sistema político democrático, a una democracia del todo inocente de su mal uso), provoca en los electores la necesidad de advertir de ese hecho a través de los escasos artilugios -el voto periódico- que generaliza para todos el modelo representativo, si se quiere, como una suerte de castigo al tenso ambiente cortoplacista que impera en la política española: repartiendo roles y dosificando la capacidad de poder político, dando incluso la menor posible en un alarde de equilibrios, para que la gestión del mismo se acompase mejor al modelo cultural de la democracia, infaustamente cada día más esquivo; avisando a la clase política para que se ubique 
en su justo sitio y sea consciente de la inherencia de la interinidad para permanecer en el poder que otorga el pueblo soberano a través de la alternancia político-electoral. Pensándolo con mesura y parsimonia, es la mejor limitación del poder que puede ejercitar la ciudadanía en el contexto del modelo partidocrático extremo inducido en la transición y cómodamente instalado desde entonces. Esta afirmación no pretende indicar que se tenga que repetir, necesariamente, este tipo de pauta en futuros comicios, pero convendría estar atentos por si acaso sucediese.

Capítulo aparte merecería, sin duda, el cúmulo de intencionados despropósitos pseudomanipulatorios, de descarado gerrymandering, que ondean alrededor del llamado voto emigrante o CERA (Censo de Electores Residentes Ausentes ${ }^{19}$ ), y que se superarán a sí mismos, con la inminente reforma del Código civil, la cual va a conseguir que Galicia, probablemente, sea el único lugar del mundo civilizado que tenga más electores que habitantes (de hecho y de derecho), acontecimiento que será, con certeza, digno de algún artículo monográfico.

${ }^{19}$ Obsérvese la contradicción, difícilmente explicable pero por sí sola indicativa, de la denominación oficial. 